\title{
Third Hankel Determinants for Subclasses of Univalent Functions
}

\author{
Paweł Zaprawa@i
}

\begin{abstract}
The main aim of this paper is to discuss the third Hankel determinants for three classes: $S^{*}$ of starlike functions, $\mathcal{K}$ of convex functions and $\mathcal{R}$ of functions whose derivative has a positive real part. Moreover, the sharp results for twofold and threefold symmetric functions from these classes are obtained.
\end{abstract}

Mathematics Subject Classification. 30C50.

Keywords. Hankel determinant, starlike functions, convex functions, $\mathrm{n}$-fold symmetric functions.

\section{Introduction}

Let $\Delta$ be the unit disk $\{z \in \mathbb{C}:|z|<1\}$ and $\mathcal{A}$ be the family of all functions $f$ analytic in $\Delta$, normalized by the condition $f(0)=f^{\prime}(0)-1=0$. It means that $f$ has the expansion $f(z)=z+\sum_{n=2}^{\infty} a_{n} z^{n}$. Pommerenke (see, $[11,12]$ ) defined the $q$-th Hankel determinant for a function $f$ as:

$$
H_{q}(n)=\left|\begin{array}{cccc}
a_{n} & a_{n+1} & \ldots & a_{n+q-1} \\
a_{n+1} & a_{n+2} & \ldots & a_{n+q} \\
\ldots & \ldots & \ldots & \ldots \\
a_{n+q-1} & a_{n+q} & \ldots & a_{n+2 q-2}
\end{array}\right|,
$$

where $n, q \in \mathbb{N}$.

In recent years, the research on Hankel determinants has focused on the estimation of $\left|H_{2}(2)\right|$. Many authors obtained results for various classes of univalent functions. It is worth citing a few of them. The exact estimates of $\left|H_{2}(2)\right|$ for the classes: $S^{*}$ of starlike functions, $\mathcal{K}$ of convex functions and $\mathcal{R}$ of functions whose derivative has a positive real part were proved by Janteng et al. [3,4]. They got the bounds: $1,1 / 8$ and $4 / 9$, respectively. For the class $S^{*}(\varphi)$ of Ma-Minda starlike functions, the exact bound of the second Hankel determinant was obtained by Lee et al. [5]. The proof of the result $\left|H_{2}(2)\right| \leq 1$ for the class $\mathcal{C}$ of close-to-convex functions can be found in the paper [15] by Selvaraj and Kumar. Other results in this direction 
are presented in $[2,7,10,16]$. On the other hand, in [19] we obtained the sharp bounds: $\left|H_{2}(2)\right| \leq 9$ and $\left|H_{2}(3)\right| \leq 15$ for the class $T$ of typically real functions.

The case $q=3$ appears to be much more difficult than the case $q=2$. Very few papers have been devoted to the third Hankel determinant. The first one was the paper by Babalola [1], who tried to estimate $\left|H_{3}(1)\right|$ for the classes $\mathcal{S}^{*}, \mathcal{K}$ and $\mathcal{R}$. Following this paper, some other authors published their results concerning $\left|H_{3}(1)\right|$ (see, for example, $[14,17,18]$ ). In [1], it was proved that

\section{Theorem 1.1.}

$$
\begin{aligned}
f \in \mathcal{S}^{*} & \Rightarrow\left|H_{3}(1)\right| \leq 16, \\
f \in \mathcal{K} & \Rightarrow\left|H_{3}(1)\right| \leq 0.714 \ldots, \\
f \in \mathcal{R} & \Rightarrow\left|H_{3}(1)\right| \leq 0.742 \ldots
\end{aligned}
$$

All results are sharp.

Moreover, Babalola claimed that the extremal functions for $\mathcal{S}^{*}$ are the rotations of $f(z)=\frac{z}{(1-z)^{2}}$. The estimates given in Theorem 1.1 are true, but rather weak, and so, not sharp! We improve these estimates in the subsequent section. There we also discuss particular subclasses of $\mathcal{S}^{*}, \mathcal{K}$ and $\mathcal{R}$ consisting of functions with so-called $n$-fold symmetry. The results for these classes, which are presented in Theorem 3.1 and in Theorem 3.3, are sharp.

It appears interesting to discuss the third Hankel determinants for functions which in particular case reduce to $f(z)=\frac{z}{(1-z)^{2}}$.

Example 1. For $f_{t}(z)=\frac{z}{1-2 z t+z^{2}}, t \in[-1,1]$, we have $f(z)=\sum_{n=1}^{\infty} U_{n-1}(t) z^{n}$. The functions $U_{n-1}(t)=\frac{\sin (n \theta)}{\sin (\theta)}, \theta=\arccos t$ are Chebyshev polynomials of the second kind. Then, applying the recurrence formula

$$
U_{n+1}(t)=2 t U_{n}(t)-U_{n-1}(t), \quad t \in[-1,1], n=1,2, \ldots,
$$

and the properties of determinants, we get

$$
H_{3}(1)=\left|\begin{array}{lll}
U_{0}(t) & U_{1}(t) & U_{2}(t) \\
U_{1}(t) & U_{2}(t) & U_{3}(t) \\
U_{2}(t) & U_{3}(t) & U_{4}(t)
\end{array}\right|=\left|\begin{array}{lll}
U_{0}(t) & U_{1}(t) & 2 t U_{1}(t)-U_{0}(t) \\
U_{1}(t) & U_{2}(t) & 2 t U_{2}(t)-U_{1}(t) \\
U_{2}(t) & U_{3}(t) & 2 t U_{3}(t)-U_{2}(t)
\end{array}\right|=0 .
$$

It is obvious that for $f_{t}, t \in[-1,1]$, this method yields that $H_{q}(n)=0$ for every positive integers $q, n$, such that $q \geq 3$.

To obtain our results, we need a few sharp estimates valid for functions with a positive real part. The class of such functions $p$ satisfying the normalization condition $p(0)=1$ is denoted by $\mathcal{P}$.

Lemma 1.1 [13]. If $p \in \mathcal{P}$, then the sharp estimate $\left|p_{n}\right| \leq 2$ holds for $n=$ $1,2, \ldots$

Lemma 1.2 [8]. If $p \in \mathcal{P}$, then the sharp estimate $\left|p_{n}-p_{k} p_{n-k}\right| \leq 2$ holds for $n, k=1,2, \ldots, n>k$.

Lemma $1.3[2]$. If $p \in \mathcal{P}$, then the sharp estimate $\left|p_{n}-\mu p_{k} p_{n-k}\right| \leq 2$ holds for $n, k=1,2, \ldots, n>k$ and $\mu \in[0,1]$. 
Lemma 1.4. If $p \in \mathcal{P}$, then the sharp estimate $\left|p_{n}-p_{k}^{2} p_{n-2 k}\right| \leq 6$ holds for $n, k=1,2, \ldots, n>2 k$.

The last lemma immediately follows from Lemmas 1.1 and 1.2. It can easily be seen when we write $p_{n}-p_{k}^{2} p_{n-2 k}=\left(p_{n}-p_{k} p_{n-k}\right)+p_{k}\left(p_{n-k}-\right.$ $\left.p_{k} p_{n-2 k}\right)$.

Moreover, Libera and Złotkiewicz proved that

Lemma 1.5 [6]. If $p \in \mathcal{P}$, then $2 p_{2}={p_{1}}^{2}+x\left(4-{p_{1}}^{2}\right)$ for some $x$ such that $|x| \leq 1$.

\section{Bounds of $\left|H_{3}(1)\right|$ for $\mathcal{S}^{*}, \mathcal{K}$ and $\mathcal{R}$}

At the beginning, observe that $H_{3}(1)$ can be written in the form

$$
H_{3}(1)=\left(a_{3} a_{5}-a_{4}^{2}\right)+a_{2}\left(a_{3} a_{4}-a_{2} a_{5}\right)+a_{3}\left(a_{2} a_{4}-a_{3}^{2}\right),
$$

or equivalently,

$$
H_{3}(1)=H_{2}(3)+a_{2} J_{2}+a_{3} H_{2}(2),
$$

where $H_{2}(k), k=2,3$ are the second Hankel determinants defined by (1) and $J_{2}=a_{3} a_{4}-a_{2} a_{5}$. The expression $J_{2}$ is a particular case of

$$
J_{n}=a_{n+1} a_{n+2}-a_{n} a_{n+3} .
$$

It seems interesting to discuss this functional in a general case for $n \in \mathbb{N}$.

As it can be seen in (1), $H_{3}(1)$ is a polynomial of four variables: $a_{2}, a_{3}$, $a_{4}, a_{5}$, where these numbers are successive coefficients of a function $f$ in a given class. However, in many cases it is possible to connect the coefficients $a_{2}, a_{3}, a_{4}, a_{5}$ with coefficients $p_{1}, p_{2}, p_{3}, p_{4}$ of a function $p \in \mathcal{P}$. To do this, we need to know the correspondence between $f$ and $p$.

Let $f, g, h$ be univalent. Then

$$
\begin{aligned}
& f \in \mathcal{S}^{*} \Leftrightarrow \frac{z f^{\prime}(z)}{f(z)} \in \mathcal{P}, \\
& g \in \mathcal{K} \Leftrightarrow 1+\frac{z g^{\prime \prime}(z)}{g^{\prime}(z)} \in \mathcal{P}, \\
& h \in \mathcal{R} \Leftrightarrow h^{\prime}(z) \in \mathcal{P} .
\end{aligned}
$$

In this section, we assume that $f(z)=z+a_{2} z^{2}+a_{3} z^{3}+\cdots, g(z)=z+$ $b_{2} z^{2}+b_{3} z^{3}+\cdots, h(z)=z+c_{2} z^{2}+c_{3} z^{3}+\cdots$ and $p(z)=1+p_{1} z+p_{2} z^{2}+\cdots$ are in $\mathcal{S}^{*}, \mathcal{K}, \mathcal{R}$ and $\mathcal{P}$, respectively.

From (8), we obtain

$$
(n-1) a_{n}=\sum_{j=1}^{n-1} a_{j} p_{n-j} .
$$

From (11), it follows that

$$
\begin{aligned}
& a_{2}=p_{1}, \quad a_{3}=\frac{1}{2}\left(p_{2}+p_{1}^{2}\right), \\
& a_{4}=\frac{1}{3}\left(p_{3}+\frac{3}{2} p_{1} p_{2}+\frac{1}{2} p_{1}^{3}\right), \quad a_{5}=\frac{1}{4}\left(p_{4}+\frac{4}{3} p_{1} p_{3}+\frac{1}{2}{p_{2}}^{2}+p_{1}^{2} p_{2}+\frac{1}{6} p_{1}^{4}\right) .
\end{aligned}
$$


Hence, for $f \in \mathcal{S}^{*}$ we have $H_{3}(1)=F\left(p_{1}, p_{2}, p_{3}, p_{4}\right)$, where

$$
\begin{aligned}
F & \left(p_{1}, p_{2}, p_{3}, p_{4}\right) \\
= & \frac{1}{144}\left[-p_{1}{ }^{6}+3 p_{1}{ }^{4} p_{2}+8 p_{1}{ }^{3} p_{3}-9 p_{1}{ }^{2} p_{2}{ }^{2}-18 p_{1}{ }^{2} p_{4}+24 p_{1} p_{2} p_{3}\right. \\
& \left.\quad-9 p_{2}{ }^{3}+18 p_{2} p_{4}-16 p_{3}{ }^{2}\right] .
\end{aligned}
$$

According to the Alexander relation, $n b_{n}=a_{n}$. Putting it into the definition of $H_{3}(1)$ for a convex function and applying the formulae (12) lead to $H_{3}(1)=G\left(p_{1}, p_{2}, p_{3}, p_{4}\right)$, where

$$
\begin{aligned}
G & \left(p_{1}, p_{2}, p_{3}, p_{4}\right) \\
= & \frac{1}{8640}\left[-p_{1}{ }^{6}+6 p_{1}{ }^{4} p_{2}+12 p_{1}{ }^{3} p_{3}-21 p_{1}{ }^{2} p_{2}{ }^{2}-36 p_{1}{ }^{2} p_{4}+36 p_{1} p_{2} p_{3}\right. \\
\quad & \left.-4 p_{2}{ }^{3}+72 p_{2} p_{4}-60 p_{3}{ }^{2}\right] .
\end{aligned}
$$

Finally, if $f \in \mathcal{R}$ then $n c_{n}=p_{n-1}$, so directly from (1) it follows that $H_{3}(1)=H\left(p_{1}, p_{2}, p_{3}, p_{4}\right)$, where

$$
H\left(p_{1}, p_{2}, p_{3}, p_{4}\right)=-\frac{1}{20} p_{1}^{2} p_{4}+\frac{1}{12} p_{1} p_{2} p_{3}-\frac{1}{27} p_{2}{ }^{3}+\frac{1}{15} p_{2} p_{4}-\frac{1}{16} p_{3}^{2} .
$$

Now, we can prove

\section{Theorem 2.1.}

$$
\begin{aligned}
& f \in \mathcal{S}^{*} \Rightarrow\left|H_{3}(1)\right| \leq 1 \\
& f \in \mathcal{K} \Rightarrow\left|H_{3}(1)\right| \leq \frac{49}{540}=0.090 \ldots \\
& f \in \mathcal{R} \Rightarrow\left|H_{3}(1)\right| \leq \frac{41}{60}=0.683 \ldots
\end{aligned}
$$

Proof. From (14),

$$
\begin{aligned}
F\left(p_{1}, p_{2}, p_{3}, p_{4}\right)= & \frac{1}{144}\left[10\left(p_{2}-{p_{1}}^{2}\right)\left(p_{4}-p_{2}{ }^{2}\right)+8\left(p_{2}-p_{1}{ }^{2}\right)\left(p_{4}-p_{1} p_{3}\right)\right. \\
& \left.+\left(p_{2}-{p_{1}}^{2}\right)^{3}-16\left(p_{3}-p_{1} p_{2}\right)^{2}\right]
\end{aligned}
$$

The triangle inequality and Lemma 1.2 lead to the declared bound for $f \in \mathcal{S}^{*}$.

If $f \in \mathcal{K}$ then, from (15),

$$
\begin{aligned}
& G\left(p_{1}, p_{2}, p_{3}, p_{4}\right)=\frac{1}{2160}\left[2\left(p_{2}-\frac{1}{2} p_{1}^{2}\right)^{3}+6 p_{4}\left(p_{2}-p_{1}^{2}\right)+9 p_{2}\left(p_{4}-p_{2}^{2}\right)\right. \\
& \left.\quad+3\left(p_{2}-p_{1}^{2}\right)\left(p_{4}-p_{1} p_{3}\right)-15 p_{3}\left(p_{3}-\frac{4}{5} p_{1} p_{2}\right)+6 p_{2}^{2}\left(p_{2}-\frac{3}{8} p_{1}^{2}\right)\right] .
\end{aligned}
$$

As above, it is enough to apply the triangle inequality and Lemmas 1.1-1.3.

In the same way, we obtain the bound for $f \in \mathcal{R}$ taking into account that

$$
\begin{aligned}
H\left(p_{1}, p_{2}, p_{3}, p_{4}\right)= & \frac{1}{20} p_{4}\left(p_{2}-{p_{1}}^{2}\right)-\frac{1}{16} p_{3}\left(p_{3}-p_{1} p_{2}\right) \\
& +\frac{1}{27} p_{2}\left(p_{4}-{p_{2}}^{2}\right)-\frac{1}{48} p_{2}\left(p_{4}-p_{1} p_{3}\right)+\frac{1}{2160} p_{2} p_{4} .
\end{aligned}
$$


An analogous calculation can be applied to obtain the result for $J_{2}$ defined by (7).

Theorem 2.2. If $f \in \mathcal{S}^{*}$ then $\left|J_{2}\right| \leq 2$.

Proof. From (12), it follows that

$$
J_{2}=\frac{1}{24}\left[p_{1}{ }^{5}+2 p_{1}{ }^{3} p_{2}-4 p_{1}{ }^{2} p_{3}+3 p_{1}{p_{2}}^{2}-6 p_{1} p_{4}+4 p_{2} p_{3}\right],
$$

and equivalently,

$$
J_{2}=\frac{1}{24}\left[\left(p_{1}^{2}-p_{2}\right)\left(p_{1}^{3}-p_{3}\right)+3\left(p_{1}^{2}-p_{2}\right)\left(p_{1} p_{2}-p_{3}\right)+6 p_{1}\left(p_{2}{ }^{2}-p_{4}\right)\right] .
$$

By Lemmas 1.1, 1.2 and $1.4,\left|J_{2}\right| \leq 2$. Moreover, equality in this estimate holds for $f(z)=\frac{z}{(1-z)^{2}}$.

From the paper [9], we know that $\left|J_{1}\right| \leq 2$ for starlike functions. Hence, it is a natural question: whether $\left|J_{n}\right| \leq 2$ for all $f \in \mathcal{S}^{*}$ and all positive integers $n$ ? Such a conjecture is supported by the fact that for the Koebe function $f(z)=\frac{z}{(1-z)^{2}}$ we have $\left|J_{n}\right|=2$ for $n=1,2, \ldots$

\section{Bounds of $\left|H_{3}(1)\right|$ for Twofold and Threefold Symmetric Functions}

Since the results in Theorem 2.1 are not sharp, it is interesting to pose a question about the magnitude of $\left|H_{3}(1)\right|$ for the discussed classes. We can give a partial answer considering functions satisfying an additional condition.

For a given class $A \subset \mathcal{A}$, a function $f \in A$ is said to be $n$-fold symmetric if $f(\varepsilon z)=\varepsilon f(z)$ holds for all $z \in \Delta$, where $\varepsilon=\exp (2 \pi i / n)$ means the principal $n$-th root of 1 . The set of all $n$-fold symmetric functions belonging to $A$ is denoted by $A^{(n)}$. If $f \in A^{(n)}$, then $f$ has the Taylor series expansion $f(z)=z+a_{n+1} z^{n+1}+a_{2 n+1} z^{2 n+1}+\cdots$. In case $n=2$, the set $A^{(2)}$ consists of all functions in $A$ which are odd.

Observe that if $f \in A^{(3)}$ then $f(z)=z+a_{4} z^{4}+a_{7} z^{7}+\cdots$, and consequently $H_{3}(1)=-a_{4}^{2}$. Similarly, if $f \in A^{(2)}$ then $f(z)=z+a_{3} z^{3}+a_{5} z^{5}+\cdots$, so $H_{3}(1)=a_{3}\left(a_{5}-a_{3}^{2}\right)$.

The definition of a $n$-fold symmetric function can be extended to functions $p$ normalized by $p(0)=1$.

\section{Theorem 3.1.}

$$
\begin{gathered}
f \in \mathcal{S}^{*(3)} \Rightarrow\left|H_{3}(1)\right| \leq \frac{4}{9}, \\
f \in \mathcal{K}^{(3)} \Rightarrow\left|H_{3}(1)\right| \leq \frac{1}{36}, \\
f \in \mathcal{R}^{(3)} \Rightarrow\left|H_{3}(1)\right| \leq \frac{1}{4} .
\end{gathered}
$$

All these bounds are sharp. 
Proof. 1. Since

$$
f \in \mathcal{S}^{*} \Leftrightarrow \sqrt[3]{f\left(z^{3}\right)} \in \mathcal{S}^{*(3)},
$$

assuming that $f(z)=z+a_{2} z^{2}+\cdots$ and $\tilde{f}(z)=\sqrt[3]{f\left(z^{3}\right)}=z+b_{4} z^{4}+\cdots$ we have $b_{4}=a_{2} / 3$. Hence, for $\tilde{f} \in \mathcal{S}^{*(3)}$,

$$
\left|H_{3}(1)\right|=\left|b_{4}\right|^{2}=\frac{1}{9}\left|a_{2}\right|^{2} \leq \frac{4}{9} .
$$

Equality holds for rotations of

$$
\tilde{f}_{0}(z)=\frac{z}{\left(1-z^{3}\right)^{2 / 3}}=z+\frac{2}{3} z^{4}+\cdots .
$$

For this function,

$$
\frac{z \tilde{f}_{0}^{\prime}(z)}{\tilde{f}_{0}(z)}=\tilde{p}_{0}(z), \quad \tilde{p}_{0}(z)=\frac{1+z^{3}}{1-z^{3}} .
$$

2. Taking into account the relation $z \tilde{g}^{\prime}(z)=\tilde{f}(z)$ valid for $\tilde{f} \in \mathcal{S}^{*(3)}$ and $\tilde{g} \in \mathcal{K}^{(3)}$, we obtain the expansion $\tilde{g}(z)=z+\frac{b_{4}}{4} z^{4}+\cdots$ and

$$
\left|H_{3}(1)\right|=\frac{1}{16}\left|b_{4}\right|^{2}=\frac{1}{144}\left|a_{2}\right|^{2} \leq \frac{1}{36},
$$

with equality for

$$
\tilde{g}_{0}(z)=\int_{0}^{z}\left(1-\zeta^{3}\right)^{-2 / 3} \mathrm{~d} \zeta=z+\frac{1}{6} z^{4}+\cdots .
$$

Obviously,

$$
1+\frac{z \tilde{g}_{0}{ }^{\prime}(z)}{\tilde{g}_{0}(z)}=\tilde{p}_{0}(z) .
$$

3. For $\tilde{h}(z)=z+c_{4} z^{4}+\cdots \in \mathcal{R}^{(3)}$ and $\tilde{p}(z)=1+p_{3} z^{3}+\cdots \in \mathcal{P}^{(3)}$, there is $4 c_{4}=p_{3}$; consequently

$$
\left|H_{3}(1)\right|=\left|c_{4}\right|^{2}=\frac{1}{16}\left|p_{3}\right|^{2} \leq \frac{1}{4} .
$$

Equality holds for

$$
\tilde{h}_{0}(z)=\int_{0}^{z} p_{0}(\zeta) \mathrm{d} \zeta=z+\frac{1}{2} z^{4}+\cdots
$$

Now, we turn to the case $n=2$. We begin with the Fekete-Szegö type functional for odd starlike functions. define

For $f(z)=z+\alpha_{3} z^{3}+\alpha_{5} z^{5}+\cdots \in A^{(2)}$ and a real number $\mu$, let us

$$
\Phi_{f}(\mu) \equiv\left|\alpha_{3}\left(\alpha_{5}-\mu \alpha_{3}^{2}\right)\right|
$$

It is clear that

$$
\left|H_{3}(1)\right|=\Phi_{f}(1)
$$


Th functional $\Phi_{f}$ is invariant under rotation. Indeed, for a given $f$ and a real $\delta$, let us define $f_{\delta}(z)=\mathrm{e}^{-i \delta} f\left(\mathrm{e}^{i \delta} z\right)$. Then, $f_{\delta}(z)=z+\alpha_{3} \mathrm{e}^{2 i \delta} z^{3}+\alpha_{5} \mathrm{e}^{4 i \delta} z^{5}+\cdots$, so

$$
\Phi_{f_{\delta}}(\mu)=\left|\mathrm{e}^{2 i \delta} \alpha_{3}\left(\mathrm{e}^{4 i \delta} \alpha_{5}-\mu \mathrm{e}^{4 i \delta} \alpha_{3}^{2}\right)\right|=\Phi_{f}(\mu) .
$$

Theorem 3.2. If $f \in \mathcal{S}^{*}(2)$, then

$$
\Phi_{f}(\mu) \leq \begin{cases}1-\mu & \mu \leq 2 / 3 \\ \frac{1}{3 \sqrt{3(2 \mu-1)}} & \mu \in[2 / 3,1] \\ \frac{1}{3 \sqrt{3(3-2 \mu)}} & \mu \in[1,4 / 3] \\ \mu-1 & \mu \geq 4 / 3 .\end{cases}
$$

The estimate is sharp.

Proof. Let $g(z)=z+a_{2} z^{2}+\cdots \in \mathcal{S}^{*}$ and $f(z)=\sqrt{g\left(z^{2}\right)}=z+\alpha_{3} z^{3}+\cdots \in$ $\mathcal{S}^{*(2)}$. Since $g \in \mathcal{S}^{*} \Leftrightarrow f \in \mathcal{S}^{*(2)}$, comparing coefficients in

$$
z^{2}+a_{2} z^{4}+a_{3} z^{6}+\cdots=\left(z+\alpha_{3} z^{3}+\alpha_{5} z^{5}+\cdots\right)^{2}
$$

we get

$$
\alpha_{3}=\frac{1}{2} a_{2}, \quad \alpha_{5}=\frac{1}{2} a_{3}-\frac{1}{8} a_{2}^{2} .
$$

Consequently, for $f \in \mathcal{S}^{*}(2)$,

$$
\Phi_{f}(\mu)=\frac{1}{4}\left|a_{2}\left(a_{3}-\frac{1+2 \mu}{4} a_{2}^{2}\right)\right| .
$$

Lemma 1.5 and (12) result in

$$
a_{3}=\frac{3}{4} p_{1}^{2}+\frac{1}{4}\left(4-{p_{1}}^{2}\right) x .
$$

Combining (12) and (28-29), we obtain

$$
\Phi_{f}(\mu)=\frac{1}{16}\left|p_{1}\left[2(1-\mu) p_{1}^{2}+\left(4-p_{1}^{2}\right) x\right]\right| .
$$

Taking into account the invariance of $\Phi_{f}$ under rotation, we can assume that $\alpha_{3}$, and so $a_{2}$ and $p_{1}$, are real. Hence, $p_{1}=2 r$ for some $r \in[0,1]$. Consequently

$$
\Phi_{f}(\mu)=\frac{1}{2} r\left|2(1-\mu) r^{2}+\left(1-r^{2}\right) x\right| .
$$

1. Suppose now that $\mu \leq 1$. Then

$$
\Phi_{f}(\mu) \leq \frac{1}{2} r\left[(1-2 \mu) r^{2}+1\right] .
$$

Let us denote by $q_{1}(r)$ the right hand side of (32).

If $\mu \leq 1 / 2$, then $q_{1}$ is increasing for $r \in[0,1]$ and so $q_{1}(r) \leq q_{1}(1)$. For $\mu \in(1 / 2,1]$, we have

$$
q_{1}(r) \leq \begin{cases}q_{1}(1) & \mu \in(1 / 2,2 / 3] \\ \left.q_{1}(1 / \sqrt{3(2 \mu-1})\right) & \mu \in[2 / 3,1] .\end{cases}
$$


2. If now $\mu \geq 1$, then

$$
\Phi_{f}(\mu) \leq \frac{1}{2} r\left[(2 \mu-3) r^{2}+1\right] .
$$

Denoting the right hand side of (33) by $q_{2}(r)$ and applying an argument similar to that given in the previous part of the proof, we obtain

$$
q_{2}(r) \leq \begin{cases}\left.q_{2}(1 / \sqrt{3(3-2 \mu})\right) & \mu \in[1,4 / 3] \\ q_{2}(1) & \mu \geq 4 / 3 .\end{cases}
$$

Finally, observe that (32) is obtained only in the case of $x=1$. From Lemma 1.5 it follows that $p_{2}=2$. Hence, equality in $(27)$ holds for $f(z)=$ $\frac{z}{1-z^{2}}$ and its rotations if $\mu \leq 2 / 3$. On the other hand, if $\mu \in[2 / 3,1]$, then the extremal functions are

$$
f(z)=\frac{z}{\left(1-z^{2}\right)^{t}\left(1+z^{2}\right)^{1-t}}, \quad t=(1+1 / \sqrt{3(2 \mu-1)}) / 2
$$

and its rotations. The Taylor series expansion of this function, in terms of $\mu$, is as follows:

$$
f(z)=z+\frac{1}{\sqrt{3(2 \mu-1)}} z^{3}+\frac{3 \mu-1}{3(2 \mu-1)} z^{5}+\cdots .
$$

Equality in (33) holds while $x=-1$. From Lemma 1.5, it follows that $p_{2}=$ $p_{1}^{2}-2$. We can deduce that if $\mu \geq 4 / 3$, then the extremal functions are rotations of $f(z)=\frac{z}{1-z^{2}}$ and if $\mu \in[1,4 / 3]$, then equality in (27) holds for

$$
f(z)=\frac{z}{\sqrt{1-2 t z^{2}+z^{4}}}, \quad t=1 / \sqrt{3(3-2 \mu)},
$$

which has the expansion in terms of $\mu$

$$
f(z)=z+\frac{1}{\sqrt{3(3-2 \mu)}} z^{3}+\frac{\mu-1}{3-2 \mu} z^{5}+\cdots,
$$

as well as its rotations.

Corollary 1. If $f \in \mathcal{S}^{*(2)}$ then $\left|\alpha_{3}\left(\alpha_{5}-\alpha_{3}^{2}\right)\right| \leq \frac{1}{3 \sqrt{3}}$. The estimate is sharp. theorem.

This and two other results for $\mathcal{K}^{(2)}$ and $\mathcal{R}^{(2)}$ are collected in the following

\section{Theorem 3.3.}

$$
\begin{gathered}
f \in \mathcal{S}^{*(2)} \Rightarrow\left|H_{3}(1)\right| \leq \frac{1}{3 \sqrt{3}}=0.192 \ldots, \\
f \in \mathcal{K}^{(2)} \Rightarrow\left|H_{3}(1)\right| \leq \frac{4}{135}=0.029 \ldots, \\
f \in \mathcal{R}^{(2)} \Rightarrow\left|H_{3}(1)\right| \leq \frac{2 \sqrt{6}}{45}=0.108 \ldots
\end{gathered}
$$

All these bounds are sharp.

Proof. 2. From equivalence $g \in \mathcal{K}^{(2)} \Leftrightarrow f(z)=z g^{\prime}(z) \in \mathcal{S}^{*(2)}$, where $f(z)=$ $z+\alpha_{3} z^{3}+\cdots, g(z)=z+\beta_{3} z^{3}+\cdots$ it follows that

$$
\beta_{3}=\frac{1}{3} \alpha_{3}, \quad \beta_{5}=\frac{1}{5} \alpha_{5},
$$


so, for $g \in \mathcal{K}^{(2)}$, there is

$$
H_{3}(1)=\left|\beta_{3}\left(\beta_{5}-\beta_{3}^{2}\right)\right|=\frac{1}{15}\left|\alpha_{3}\left(\alpha_{5}-\frac{5}{9} \alpha_{3}^{2}\right)\right| .
$$

Applying Theorem 3.2, we obtain the claimed bound.

3. Let $h(z)=z+\gamma_{3} z^{3}+\cdots \in \mathcal{R}^{(2)}$. Since $h \in \mathcal{R}^{(2)} \Leftrightarrow h^{\prime} \in \mathcal{P}^{(2)}$, we can write $h^{\prime}(z)=\frac{z f^{\prime}(z)}{f(z)}$ for some $f \in \mathcal{S}^{*(2)}$. Comparing coefficients in

$$
\left(1+3 \gamma_{3} z^{2}+5 \gamma_{5} z^{4} \ldots\right)\left(z+\alpha_{3} z^{3}+\alpha_{5} z^{5}+\cdots\right)=z+3 \alpha_{3} z^{3}+5 \alpha_{5} z^{5}+\cdots
$$

leads to

$$
\gamma_{3}=\frac{2}{3} \alpha_{3}, \quad \gamma_{5}=\frac{4}{5} \alpha_{5}-\frac{2}{5} \alpha_{3}^{2}
$$

Hence, for $h \in \mathcal{R}^{(2)}$,

$$
H_{3}(1)=\left|\gamma_{3}\left(\gamma_{5}-{\gamma_{3}}^{2}\right)\right|=\frac{8}{15}\left|\alpha_{3}\left(\alpha_{5}-\frac{19}{18} \alpha_{3}^{2}\right)\right| .
$$

Once again, from Theorem 3.2, we get the desired bound. The sharpness of all these estimates follows from Theorem 3.2.

Open Access. This article is distributed under the terms of the Creative Commons Attribution 4.0 International License (http://creativecommons.org/licenses/ by/4.0/), which permits unrestricted use, distribution, and reproduction in any medium, provided you give appropriate credit to the original author(s) and the source, provide a link to the Creative Commons license, and indicate if changes were made.

\section{References}

[1] Babalola, K.O.: On $H_{3}(1)$ Hankel determinants for some classes of univalent functions. In: Dragomir, S.S., Cho, J.Y. (eds.) Inequality Theory and Applications, vol. 6, pp. 1-7. Nova Science Publishers, New York (2010)

[2] Hayami, T., Owa, S.: Generalized Hankel determinant for certain classes. Int. J. Math. Anal. 4(52), 2573-2585 (2010)

[3] Janteng, A., Halim, S.A., Darus, M.: Coefficient inequality for a function whose derivative has a positive real part. J. Inequal. Pure Appl. Math. 7(2), 1-5 (2006)

[4] Janteng, A., Halim, S.A., Darus, M.: Hankel determinant for starlike and convex functions. Int. J. Math. Anal. 1(13), 619-625 (2007)

[5] Lee, S.K., Ravichandran, V., Supramaniam, S.: Bounds for the second Hankel determinant of certain univalent functions. J. Inequal. Appl. (2013). Art. 281. doi:10.1186/1029-242X-2013-281

[6] Libera, R.J., Zotkiewicz, E.J.: Early coefficients of the inverse of a regular convex function. Proc. Am. Math. Soc. 85, 225-230 (1982)

[7] Liu, M.S., Xu, J.F., Yang, M.: Upper bound of second Hankel determinant for certain subclasses of analytic functions. Abstr. Appl Anal. (2014). Art. 603180. doi:10.1155/2014/603180

[8] Livingston, A.E.: The coefficients of multivalent close-to-convex functions. Proc. Am. Math. Soc. 21, 545-552 (1969) 
[9] Ma, W.: Generalized Zalcman conjecture for starlike and typically real functions. J. Math. Anal. Appl. 234(1), 328-339 (1999)

[10] Noonan, J.W., Thomas, D.K.: On the Hankel determinants of areally mean p-valent functions. Proc. Lond. Math. Soc. 3(25), 503-524 (1972)

[11] Pommerenke, C.: On the coefficients and Hankel determinants of univalent functions. Proc. Lond. Math. Soc. 3(41), 111-122 (1966)

[12] Pommerenke, C.: On the Hankel determinants of univalent functions. Mathematika 14, 108-112 (1967)

[13] Pommerenke, C.: Univalent functions. Vandenhoeck and Ruprecht, Göttingen (1975)

[14] Raza, M., Malik, S.N.: Upper bound of third Hankel determinant for a class of analytic functions related with lemniscate of Bernoulli. J Inequal. Appl. (2013). Art. 412. doi:10.1186/1029-242X-2013-412

[15] Selvaraj, C., Kumar, T.R.K.: Second Hankel determinant for certain classes of analytic functions. Int. J. Appl. Math. 28(1), 37-50 (2015)

[16] Vamshee Krishna, D., RamReddy, T.: Hankel determinant for starlike and convex functions of order alpha. Tbil. Math. J. 5, 65-76 (2012)

[17] Vamshee Krishna, D., Venkateswarlua, B., RamReddy, T.: Third Hankel determinant for bounded turning functions of order alpha. J. Niger. Math. Soc. 34, 121-127 (2015)

[18] Vamshee Krishna, D., Venkateswarlua, B., RamReddy, T.: Third Hankel determinant for certain subclass of p-valent functions. Complex Var. Elliptic Equ. (2015). Available from http://dx.doi.org/10.1080/17476933.2015.1012162

[19] Zaprawa, P.: Second Hankel determinants for the class of typically real functions. Abstr. Appl. Anal. (2016). Art. 3792367. doi:10.1155/2016/3792367

Pawel Zaprawa

Department of Mathematics

Faculty of Mechanical Engineering

Lublin University of Technology

Nadbystrzycka 38D

20-618 Lublin

Poland

e-mail: p.zaprawa@pollub.pl

Received: February 4, 2016.

Revised: November 9, 2016.

Accepted: November 24, 2016. 